\title{
Evaluation of the macula, retinal nerve fiber layer and choroid thickness in postmenopausal women and reproductive-age women using spectral-domain optical coherence tomography
}

\author{
Mustafa Ataş ${ }^{1}$, Gökhan Açmaz ${ }^{2}$, Hüseyin Aksoy³, Süleyman Demircan ${ }^{1}$, Altan Göktaş ${ }^{1}$, \\ Hasan Basri Arifoğlu, Gökmen Zararsız ${ }^{4}$ \\ ${ }^{1}$ Department of Ophthalmology, Kayseri Education and Research Hospital, Kayseri, Turkey \\ Department of Obstetrics and Gynecology, Kayseri Education and Research Hospital, Kayseri, Turkey \\ ${ }^{3}$ Department of Obstetrics and Gynecology, Kayseri Military Hospital, Kayseri, Turkey \\ ${ }^{4}$ Department of Statistics, Erciyes University School of Medicine, Kayseri, Turkey
}

\section{Abstract}

Objective: Menopause is a physiological life period that potentially affects various organs and systems. Therefore, a wide spectrum of signs and symptoms of eyes may be clinically observed in the postmenopausal period.

Aim of the study: To evaluate the macular, retinal nerve fiber layer (RNFL) and choroidal thickness alterations by using spectral-domain optical coherence tomography (SD-OCT) in postmenopausal women and compare with healthy reproductive-age women controls.

Material and methods: The study population included a healthy reproductive-age control group $(n=72)$ and postmenopausal study group $(n=72)$. Retinal thickness parameters were measured by SD-OCT. Peripapillary RNFL thickness parameters, macular thickness and choroidal thickness were evaluated.

Results: Superior inner macula, temporal inner macula, inferior inner macula, nasal inner macula, inferior outer macula and choroid thickness were significantly thinner in the postmenopausal study group than the healthy reproductive-age control group $(p=0.007, p=0.037, p=0.027, p=0.006, p=0.016, p<0.001$, respectively). After adjusting for age, only choroid thickness was significantly thinner in the postmenopausal study group than controls $\left(p^{\dagger}=0.005\right)$.

Conclusions: This study revealed that choroidal thickness measured by SD-OCT was significantly thinner in postmenopausal women than healthy reproductive-age women. We can speculate that the decrease in choroidal thickness in postmenopausal women may indicate a reduced estrogen-dependent vasodilatory effect in ophthalmic artery secondary to menopausal estrogen deficiency.

Key words: macula, retinal nerve fiber layer, choroid, menopause, optical coherence tomography.

\section{Introduction}

Simultaneously with success in postponing death, the ratio of the elderly population is rapidly increasing. This increase in the elderly population is also increasing the rate of postmenopausal women in society. Despite a great increase in the life expectancy of women, the age at menopause has remained remarkably constant. As the proportion of older females in the population increases, an increasing prevalence of menopause and age-related disease may be expected to be seen [1]. Menopause is also associated with ocular changes. An increase in the incidence of ocular pathologies and ocular symptoms have been observed during the postmenopausal period. Postmenopausal hormonal status also seems to play a role in these ocular pathologies [2].

For decades, conventional imaging methods with low resolution and low accuracy rate have been used to evaluate the ocular changes in postmenopausal women. Moreover, interobserver conflicts often exist for previous ocular imaging techniques. Optical coherence tomography (OCT) is a new diagnostic imaging modality that provides high-resolution, cross-sectional images of ocular structures with a longitudinal resolution of 3-10 $\mu \mathrm{m}$ [3]. This provides high-resolution ocular images in vivo when compared with other available imaging techniques. Optical coherence tomography makes it possible to obtain noninvasive, rapid, objective, high-resolution, cross-sectional imaging of the ret- 
ina and also permits direct, real-time imaging of ocular pathology that previously could not be visualized using traditional methods. There is no recent comprehensive study using OCT which investigates the influence of menopause on the retinal structure. To our knowledge, the present study in which macular, RNFL and choroidal thickness measured together using OCT is the first such research reported in the literature.

The aim of the present study was to evaluate the macular, choroid and peripapillary nerve fiber layer thickness in postmenopausal women by means of OCT and to compare these findings in healthy reproductiveage controls.

\section{Material and methods}

The study was approved by the institutional ethics committee (no. 2013/60) and all participants signed an informed consent form regarding participation in the present study. This prospective study was performed at the Kayseri Education and Training Hospital of Medicine, a tertiary referral centre in Turkey between January 2013 and May 2013. The study was carried out with two groups. The study group comprised 72 postmenopausal women and the control group comprised 72 healthy women volunteers at the reproductive age. Postmenopausal women were at least 1 year after spontaneous menopause in association with elevated FSH serum levels (> $40 \mathrm{IU} / \mathrm{L}$ ). None of the women in the control group was pregnant.
Women who reported histories of any medical problems, taking hormone replacement therapy (especially six months prior to the study or current use of hormone replacement therapy [HRT]), acute or chronic liver diseases, kidney and pulmonary diseases were excluded from the study. Subjects were also excluded if they used caffeine or tobacco. Furthermore, ocular surgery, ocular trauma or any ocular disease at the time of OCT measurement were also excluded from the study.

Each subject underwent a comprehensive ophthalmologic examination. Following this detailed ophthalmologic examination, the third-generation Spectralis OCT device (software version 5.6.3.0; Spectralis OCT Heidelberg Engineering, Dossenheim, Germany) was used for the assessment. The OCT assessments of subjects involved in the study were performed by the same experienced technician. The procedure was achieved without pupillary dilatation and under the same intensity of dim room lighting.

Macular thickness was determined automatically and was analyzed by OCT software. Fast Macular Thickness Map option was utilized for the macular measurements. During the assessments, macular thickness and volume analysis were used. The results obtained from the macular scan were classified by region as shown in Figure 1. We selected the macular map analysis protocol (Fig. 2) on the Spectralis to display numeric averages of the measurements for each of 9 subfields as defined by the Early Treatment Diabetic Retinopathy Study (ETDRS) [4].

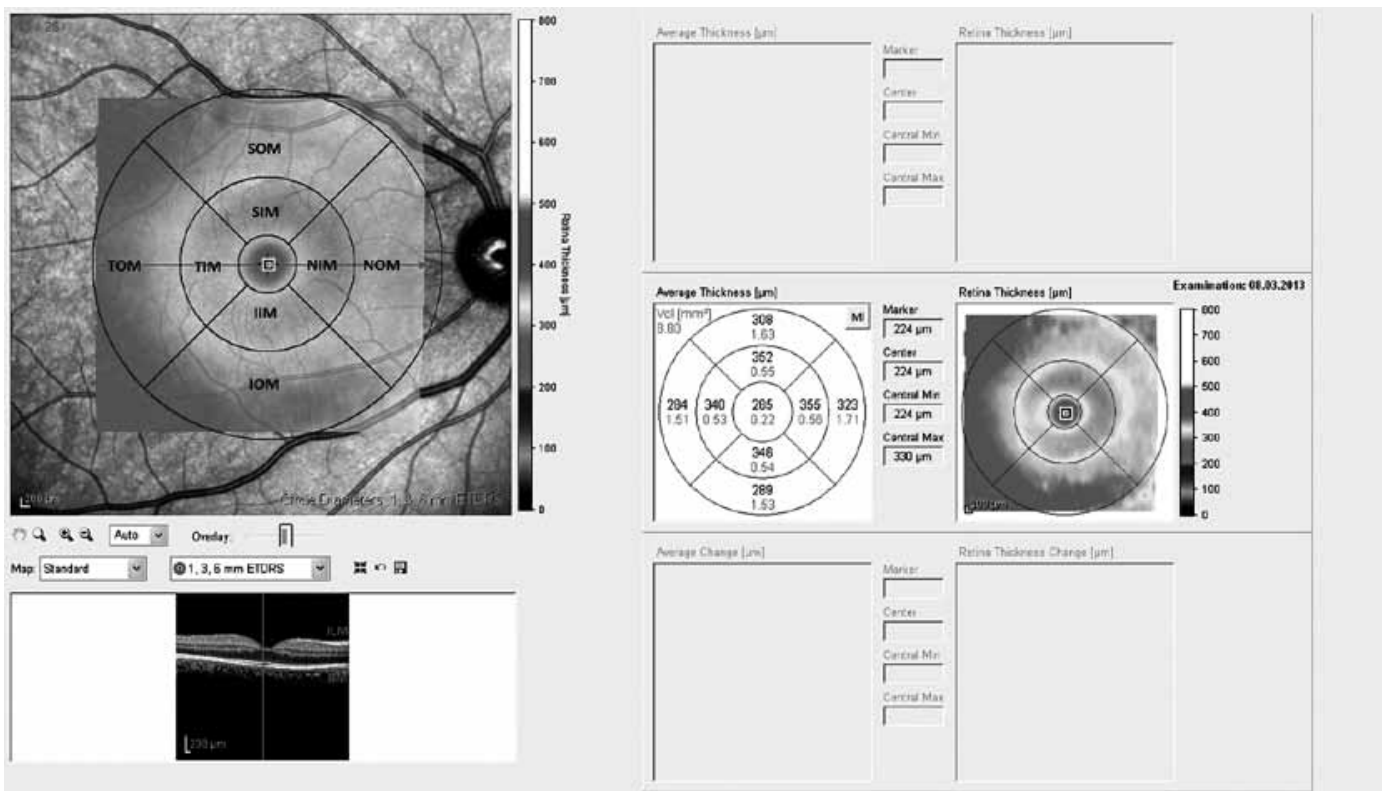

CSF - central subfield, IIM - inferior inner macula, IOM - inferior outer macula, NIM - nasal inner macula, NOM - nasal outer macula, SIM - superior inner macula, SOM - superior outer macula, TIM - temporal inner macula, TOM - temporal outer macula

Fig. 1. Early Treatment Diabetic Retinopathy Study subfield template used to derive macular thickness measurements from different regions of a high-density volume scan of the macula. Circles are 1, 3, and $6 \mathrm{~mm}$ in diameter 

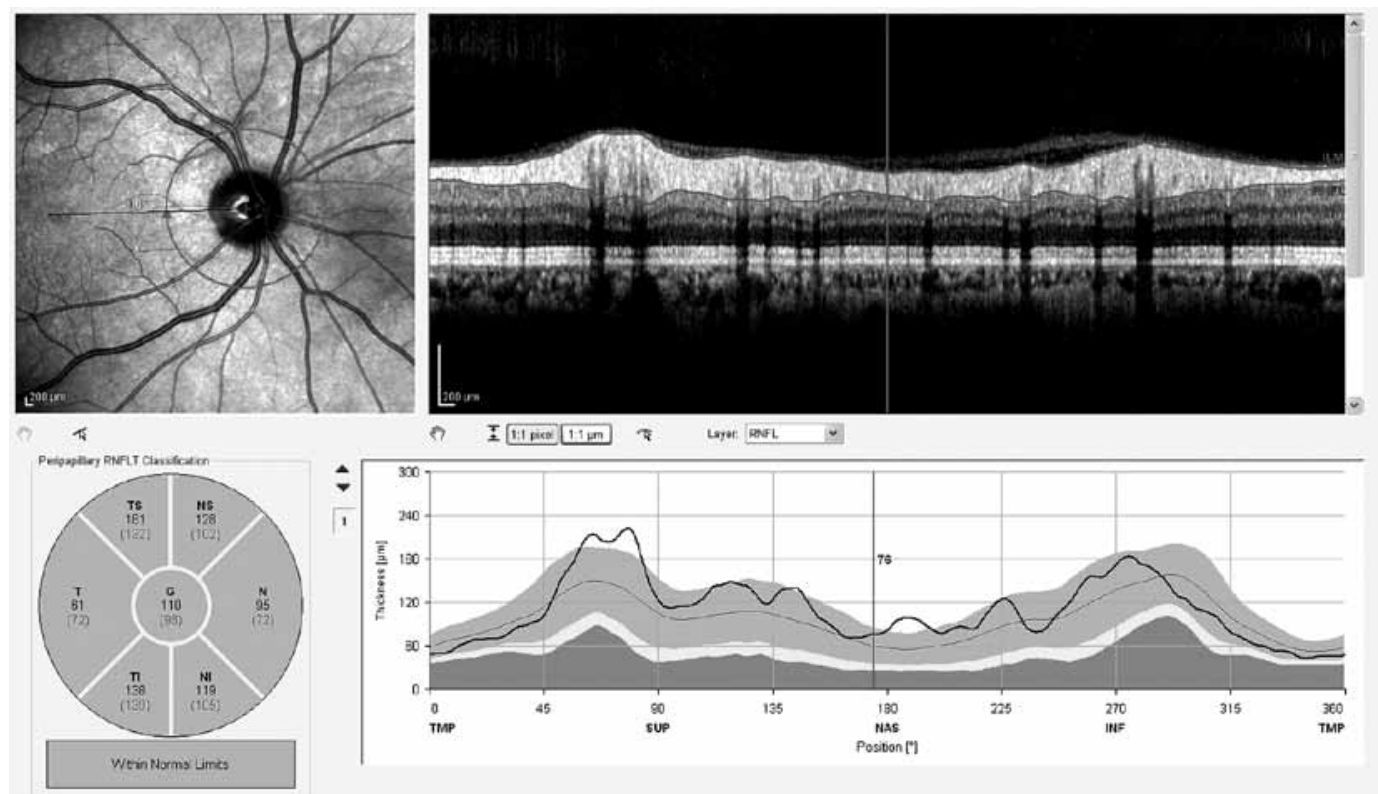

Ts - temporal superior, $\mathrm{Ns}$ - nasal superior, $\mathrm{N}$ - nasal, $\mathrm{Ni}$ - nasal inferior, $\mathrm{Ti}$ - temporal inferior, $\mathrm{G}$ - average

Fig. 2. Sample RNFL report provided by SD-OCT. Numbers reflect individual mean RNFL thickness. Numbers in parentheses are the mean thickness of adults in the Spectralis normative database

The peripapillary RNFL thickness parameters that were automatically calculated by the fast RNFL mode and divided into regions included temporal quadrant thickness, temporal superior quadrant thickness, nasal superior quadrant thickness, nasal quadrant thickness, nasal inferior quadrant thickness, temporal inferior quadrant thickness, and average thickness. The method of obtaining enhanced depth imaging (EDI) OCT images has been reported previously [5]. The resultant images were viewed and measured with the contained
Heidelberg Eye Explorer software (version 5.6.3.0; Heidelberg Engineering). Choroid imaging was obtained by activating the EDI button. The choroid was manually measured from the outer border of the hyper-reflective line corresponding to the retinal pigment epithelium to the inner surface of the sclera (Fig. 3). The measurements of subfoveal choroidal thickness were done by one of the authors in a masked fashion without knowledge of subject information.

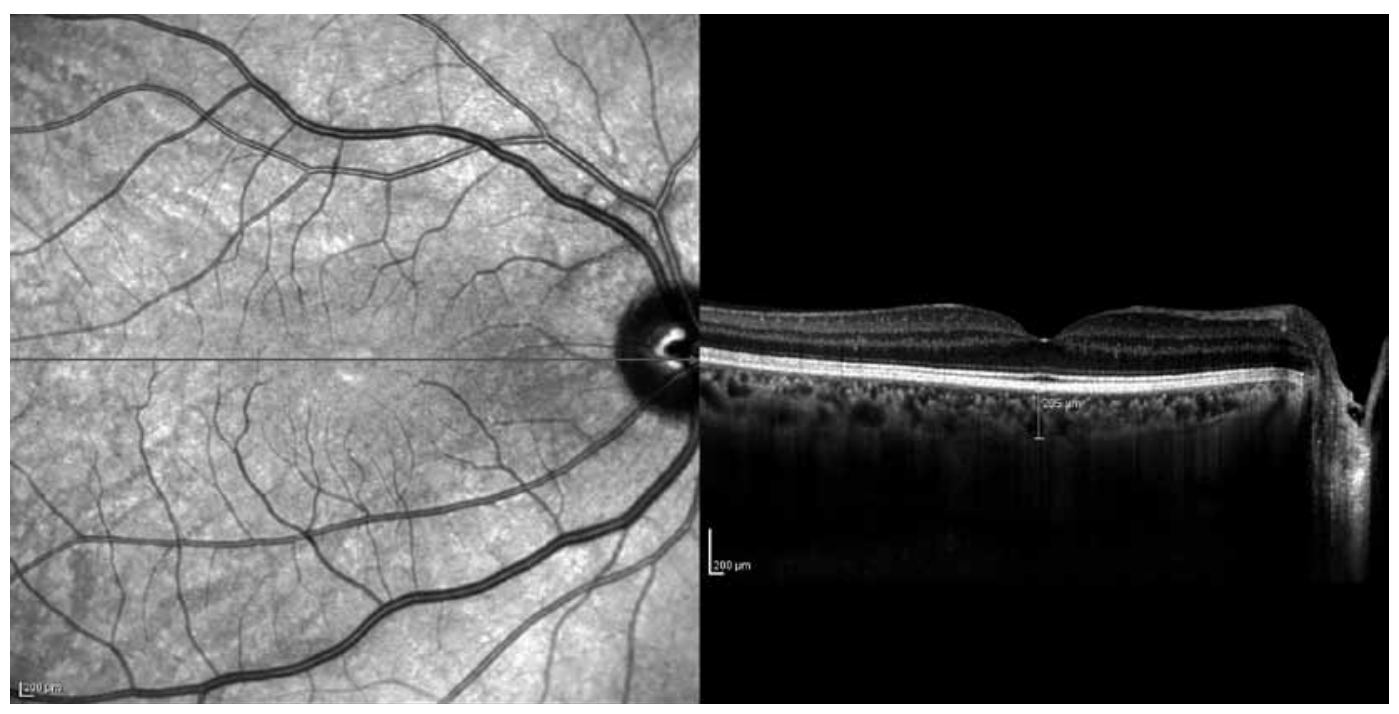

Fig. 3. Measurement of subfoveal choroidal thickness with EDI with Spectralis OCT 


\section{Statistical analysis}

Statistical analysis was conducted using R 3.0.0 software. Shapiro-Wilk's test was used, histogram and q-q plot were examined to assess the data normality. Levene test was used to assess the variance homogeneity. A two-sided independent samples $t$ test was applied to compare the differences of variables between groups. One-way analysis of covariance (ANCOVA) was also applied to compare the differences of variables between groups by adjusting the age variable. Values are expressed as mean \pm standard deviation. $p<0.05$ value was considered as statistically significant.

\section{Results}

The study group included 72 eyes of 72 postmenopausal women, control group included 72 eyes of 72 healthy reproductive-age women. Mean age was $56.89 \pm 4.63$ and $39.57 \pm 8.41$ in study and control groups, respectively. There was a statistical difference for age between the study group and healthy reproductive-age controls $(p<0.001)$.

None of the patients had retinopathy, retinal hemorrhages, optical papilledema, retinal or macular edema at the time of examination. The macular and choroidal thickness analysis, macular volume analysis, and peripapillary RNFL thickness analysis obtained by the SDOCT were displayed in Table I, Table II, and Table III, respectively.

SIM, TIM, IIM, NIM, IOM and choroid thickness were significantly thinner in the postmenopausal study

Table I. Average macular and choroidal thickness among postmenopausal women and healthy reproductive-age women

\begin{tabular}{|c|c|c|c|c|}
\hline Variables & $\begin{array}{l}\text { Control group } \\
\quad(n=72)\end{array}$ & $\begin{array}{l}\text { Postmeno- } \\
\text { pause group } \\
(n=72)\end{array}$ & $p$ & $p^{+}$ \\
\hline CSF & $264.93 \pm 22.15$ & $263.01 \pm 18.45$ & 0.574 & 0.042 \\
\hline SIM & $342.83 \pm 12.76$ & $335.13 \pm 20.16$ & 0.007 & 0.354 \\
\hline TIM & $334.47 \pm 12.57$ & $328.90 \pm 18.57$ & 0.037 & 0.056 \\
\hline$\| M$ & $336.51 \pm 13.18$ & $330.49 \pm 18.67$ & 0.027 & 0.286 \\
\hline NIM & $334.93 \pm 13.90$ & $327.88 \pm 16.35$ & 0.006 & 0.266 \\
\hline SOM & $295.79 \pm 15.79$ & $291.92 \pm 20.41$ & 0.205 & 0.675 \\
\hline TOM & $299.29 \pm 23.44$ & $293.43 \pm 19.77$ & 0.107 & 0.636 \\
\hline IOM & $290.96 \pm 16.21$ & $284.63 \pm 14.81$ & 0.016 & 0.546 \\
\hline NOM & $297.42 \pm 23.36$ & $289.99 \pm 22.13$ & 0.052 & 0.821 \\
\hline $\begin{array}{l}\text { Foveal } \\
\text { center }\end{array}$ & $224.36 \pm 30.79$ & $226.86 \pm 23.4$ & 0.584 & 0.202 \\
\hline Choroid & $325.44 \pm 65.75$ & $271.3 \pm 65.63$ & $<0.001$ & 0.005 \\
\hline \multicolumn{5}{|c|}{$\begin{array}{l}\text { CSF - central subfield, IIM - inferior inner macula, IOM - inferior outer } \\
\text { macula, NIM - nasal inner macula, NOM - nasal outer macula, SIM - } \\
\text { superior inner macula, SOM - superior outer macula, TIM - temporal inner } \\
\text { macula, TOM - temporal outer macula } \\
\text { Values are expressed as mean } \pm \text { SD } \\
p^{\dagger}-\text { age-adjusted } p \text { value }\end{array}$} \\
\hline
\end{tabular}

group than the healthy reproductive-age control group $(p=0.007, p=0.037, p=0.027, p=0.006, p=0.016$, $p<0.001$, respectively). After adjusting for age only choroid thickness was significantly thinner in the postmenopausal study group than controls $\left(p^{+}=0.005\right)$. SIM, TOM, IOM and NOM volumes were significantly thinner in the postmenopausal study group than healthy reproductive-age controls $(p=0.013, p=0.029, p=0.012$, $p=0.039$, respectively). However after adjusting for age there was no statistically significant difference between postmenopausal study and control groups for macular volume parameters.

No significant difference was found between the postmenopausal study and control groups regarding all the peripapillary RNFL thickness parameters.

Table II. Average macular volume among postmenopausal women and healthy reproductive-age women

\begin{tabular}{lcccc}
\hline Variables & $\begin{array}{c}\text { Control group } \\
(n=72)\end{array}$ & $\begin{array}{c}\text { Postmeno- } \\
\text { pause group } \\
(n=72)\end{array}$ & $p$ & $p^{\dagger}$ \\
\hline CSF & $0.21 \pm 0.02$ & $0.21 \pm 0.01$ & 0.678 & 0.026 \\
\hline SIM & $0.54 \pm 0.02$ & $0.53 \pm 0.02$ & 0.013 & 0.314 \\
\hline TIM & $0.53 \pm 0.02$ & $0.52 \pm 0.02$ & 0.172 & 0.364 \\
\hline IIM & $0.53 \pm 0.02$ & $0.52 \pm 0.02$ & 0.054 & 0.744 \\
\hline NIM & $0.53 \pm 0.02$ & $0.54 \pm 0.18$ & 0.383 & 0.637 \\
\hline SOM & $1.58 \pm 0.08$ & $1.55 \pm 0.10$ & 0.073 & 0.647 \\
\hline TOM & $1.59 \pm 0.11$ & $1.55 \pm 0.11$ & 0.029 & 0.426 \\
\hline IOM & $1.54 \pm 0.09$ & $1.51 \pm 0.08$ & 0.012 & 0.558 \\
\hline NOM & $1.58 \pm 0.12$ & $1.54 \pm 0.11$ & 0.039 & 0.788 \\
\hline Total & $8.62 \pm 0.33$ & $8.46 \pm 0.38$ & 0.627 & 0.631 \\
volume & & &
\end{tabular}

CSF - central subfield, IIM - inferior inner macula, IOM - inferior outer macula, NIM - nasal inner macula, NOM - nasal outer macula, SIM superior inner macula, SOM - superior outer macula, TIM - temporal inner macula, TOM - temporal outer macula

Values are expressed as mean \pm SD

$p^{\dagger}-$ age-adjusted $p$ value

Table III. Average peripapillary RNFL thickness among postmenopausal women and healthy reproductive-age women

\begin{tabular}{lcccc}
\hline Variables & $\begin{array}{c}\text { Control group } \\
(n=72)\end{array}$ & $\begin{array}{c}\text { Postmeno- } \\
\text { pause group } \\
(n=72)\end{array}$ & $p$ & $p^{\dagger}$ \\
\hline Temporal & $70.19 \pm 12.88$ & $69.41 \pm 11.59$ & 0.703 & 0.738 \\
\hline $\mathrm{Ts}$ & $136.74 \pm 22.45$ & $133.78 \pm 19.25$ & 0.404 & 0.347 \\
\hline $\mathrm{Ns}$ & $111.31 \pm 22.44$ & $116.25 \pm 22.74$ & 0.196 & 0.153 \\
\hline $\mathrm{N}$ & $75.61 \pm 15.68$ & $74.16 \pm 11.29$ & 0.531 & 0.364 \\
\hline $\mathrm{Ni}$ & $114.43 \pm 25.39$ & $118.07 \pm 25.26$ & 0.395 & 0.188 \\
\hline $\mathrm{Ti}$ & $143.49 \pm 26.41$ & $140.09 \pm 17.66$ & 0.369 & 0.351 \\
\hline $\mathrm{G}$ & $99.65 \pm 12.18$ & $99.58 \pm 8.88$ & 0.968 & 0.088
\end{tabular}

Ts - temporal superior, Ns - nasal superior, $\mathrm{N}$ - nasal, $\mathrm{Ni}$ - nasal inferior, $\mathrm{Ti}$ - temporal inferior, $\mathrm{G}$ - average Values are expressed as mean \pm SD $p^{\dagger}-$ age-adjusted $p$ value 


\section{Discussion}

Due to the increase in life expectancy and the constant age at menopause, women can now expect to spend more than one-third of their lives in the postmenopausal period. Increases in life expectancies mean that women are spending longer periods of their life in a hypo-estrogenic state. Understanding the potential effects of menopause on various organs and systems and long-term consequences of sex hormones deficiency secondary to menopause requires long-term data on numerous postmenopausal women from different backgrounds [1]. In the postmenopausal period, many organs and systems of the body are susceptible to postmenopausal sex hormones deficiency. Therefore, a wide spectrum of signs and symptoms associated with many organs may be clinically observed in menopause. Eye is one of the organs affected by menopause. Thus, menopause may cause major ocular changes [6].

Due to the use of conventional imaging methods, the diagnosis and follow-up of retinal disease are problematic. Until recently, traditional imaging modalities have been used to evaluate the ocular findings of menopause [7]. However, there are important limitations of previous imaging methods such as lower image resolution, low measurement accuracy and interobserver conflicts. Therefore, an objective, quantitative, and high sensitive imaging modality to assess retinal structure is needed for diagnosing and follow-up of retinal disease.

Optical coherence tomography is a noninvasive, noncontact, transpupillary imaging modality that has been recently introduced into clinical practice. It is analogous to B-scan ultrasonography, but instead of sound waves, it uses light waves to obtain high-resolution cross-sectional images of the retina [5]. This new technological advance in ophthalmologic imaging provides quantitative measurement or morphometry of the retinal structure. Quantitative and detailed each ocular layer thickness is an important early diagnostic sign for eye evaluation. Compared with the available visual imaging techniques, OCT provides several technical improvements, including higher resolution and scan speed, improved image segmentation, and better measurement reproducibility, which may increase its clinical usefulness for detection of several retinal diseases [8]. Optical coherence tomography is currently the most precise method to measure retinal thickness in vivo. This new medical imaging technology is increasingly used in the diagnosis and monitoring of retinal diseases including age-related macular degeneration, diabetic retinopathy, and glaucoma. The anatomic layers within the retina can be differentiated and retinal thickness can be measured at the micrometer level with a resolution of 3-10 $\mu \mathrm{m}$ [9]. Recently, the introduction of a new version of this technology, SD-OCT, has greatly enhanced the resolution and decreased scan acquisition times compared to TD-OCT [10]. This new technological advance in ophthalmologic imaging provides histologic-like cross-sectional images of the vitreoretinal interface and the retinal structures [11].

In vivo qualitative and quantitative imaging of the retina by OCT is noninvasive, obtainable, reproducible and reliable diagnostic modality in both healthy subjects and in patients with retinal pathology [12]. This new imaging modality was also used in the evaluation of age-related retinal diseases and their management.

Estrogen receptors have quite recently been demonstrated in various ocular tissues, such as the conjunctiva, cornea, meibomian gland, choroid, retina, and lens [13]. Because of the presence of sex steroid hormone receptors in various ocular tissues, sex steroid hormones may have a significant effect on ocular physiology and structure. Since the major consequences of menopause are related primarily to sex steroid hormones deficiency, menopause may significantly impact the ocular structure and function thereby reducing the quality of life [14]. The accurate assessment of ocular findings in menopause is crucial for a better clinical understanding of retinal changes of menopause, but adequate visualization of the retinal tissue using OCT has not been possible until recently.

In a study Ciccone et al. showed that administration of single dose nasal 17- $\beta$-estradiol increases ophthalmic artery perfusion [15]. On the other hand, Atalay et al. investigated ocular hemodynamic parameters using Doppler. They concluded that hormone replacement therapy with estradiol 17-valerate $2 \mathrm{mg}$ plus cyproterone acetate $1 \mathrm{mg}$ improves ocular vascular Doppler indices which may be a reflection of cerebral vascular status [16]. We can speculate that the decrease in choroidal thickness in postmenopausal women may indicate a reduced estrogen-dependent vasodilatory effect in ophthalmic artery secondary to menopausal estrogen deficiency. Hormonal changes may affect many of the organs and systems. Such as it has been shown it impaired occupational functioning, quality of life, selfesteem, life satisfaction, femininity, anxiety, depression found to be related with hormonal changes [17-23]. In addition to hormonal changes in women during menopause, excessive weight gain is a common phenomenon. It leads to the development of abdominal obesity, which together with co-occurring insulin resistance, dyslipidemia and hypertension forms a menopausal metabolic syndrome. This situation may lead to both increase in the intensity of atheromatous lesions in coronary arteries [18] and nocturnal decline in blood pressure. Moreover for women, the menopause stage is connected with chronic diseases. There is no doubt that the age of the patient is a factor strongly correlated with the possibility of ocular operations. It has been shown that ocular operations may affect ocular circulation [19]. Therefore, we are of the opinion that 
thinner choroid may be related with previous operations, cardio-vascular and hormonal changes in postmenopausal women.

There is no recent comprehensive study using OCT which investigates the influence of menopause on the retinal structure. In our study, postmenopausal women and healthy reproductive-age women controls were used together in the same study. We noted that choroidal thickness was significantly thinner in postmenopausal women compared to healthy reproductiveage controls. The strength of this study was the use of current commercially available SD-OCT technology. The main weakness of our study was the difference of age between the study group and controls. This difference was statistically significant but it was difficult to find age-matched reproductive women controls with the study group.

In conclusion, this study revealed that choroidal thickness measured by SD-OCT was significantly thinner in postmenopausal women than healthy reproductive-age women.

\section{Acknowledgements}

We thank Dr. Faruk Balkaya (English Department of Kayseri Erciyes University) for his contribution to English terms and language.

\section{Disclosure}

This study was conducted in the Kayseri Education and Research Hospital.

The Kayseri Education and Research Hospital financially supported this study.

Authors report no conflicts of interest.

\section{References}

1. Soules M, Herman S, Parrott E, et al. Executive summary: stages of reproductive aging workshop (STRAW). Fertil Streil 2001; 76: 874-878.

2. Metka M, Enzelsberger $H$, Knogler W, et al. Ophthalmic complaints as a climacteric symptom. Maturitas 1991; 14: 3-8.

3. Huang D, Swanson EA, Lin CP, et al. Optical coherence tomography. Science 1991; 254: 1178-1181.

4. Early Treatment Diabetic Retinopathy Study Research Group. ETDRS Report No. 7: Early Treatment Diabetic Retinopathy Study design and baseline patient characteristics. Ophthalmology 1991; 98: 741-756.

5. Baumal CR. Clinical applications of ocular coherence tomography. Curr Opin Ophthalmol 1999; 101: 182-188.

6. Ogueta SB, Schwartz SD, Yamashita CK, et al. Estrogen receptor in the human eye: influence of gender and age on gene expression. Invest Ophthalmol Vis Sci 1999; 40: 1906-1911.

7. Faria AF, de Souza MA, Geber S. Vascular resistance of central retinal artery is reduced in postmenopausal women after use of estrogen. Menopause 2011; 18: 869-872.

8. Povazay B, Hofer B,Torti C, et al. Impact of enhanced resolution, speed and penetration on three-dimensional retinal optical coherence tomography. Opt Exp 2009; 17: 4134-4150.

9. Budenz DL, Chang RT, Huang X, et al. Reproducibility of retinal nerve fiber thickness measurements using the Stratus OCT in normal and glaucomatous eyes. Invest Ophthalmol Vis Sci 2005; 46: 2440-2443.

10. Wojtkowski M, Srinivasan V, Fujimoto JG, et al. Threedimensional retinal imaging with high-speed ultrahigh-resolution optical coherence tomography. Ophthalmology 2005; 112: 1734-1746.

11. Regatieri CV, Branchini L, Fujimoto JG, et al. Choroidal imaging using spectral-domain optical coherence tomography. Retina 2012; 32: 865-876.

12. Van Velthoven ME, Faber DJ, Verbraak FD, et al. Recent developments in optical coherence tomography for imaging the retina. Prog Retin Eye Res 2007; 26: 57-77.

13. Fuchsjager-Mayrl G, Nepp J, Schneeberger C, et al. Identification of estrogen and progesterone receptor mRNA expression in the conjunctiva of premenopausal women. Invest Ophthalmol Vis Sci 2002; 43: 2841-2844.

14. Siesky BA, Harris A, Patel C, et al. Comparison of visual function and ocular hemodynamics between pre- and post-menopausal women. Eur J Ophthalmol 2008; 18: 320-323.

15. Ciccone MM, Cicinelli E, Giovanni A, et al. Ophthalmic artery vasodilation after intranasal estradiol use in postmenopausal women. J Atheroscler Thromb 2012; 19: 1061-1065.

16. Atalay E, Karaali K, Akar M, et al. Early impact of hormone replacement therapy on vascular hemodynamics detected via ocular colour Doppler analysis. Maturitas 2005; 50: 282-288.

17. Açmaz G, Albayrak E, Acmaz B, et al. Level of Anxiety, Depression, SelfEsteem, Social Anxiety, and Quality of Life among the Women with Polycystic Ovary Syndrome. The Scientific World Journal; vol. 2013; http:// dx.doi.org/10.1155/2013/851815.

18. Zwoliński R, Ostrowski S, Walczak A, et al. Long-term results of coronary artery bypass grafting in women under 45 years of age. Kardiochir Torakochir Pol 2012; 1: 22-27.

19. Stopa M, Kocięcki J, Rakowicz P, Dmitriew A. Case report A pedicled autologous choroid RPE patch: a technique to preserve perfusion. Videosurgery Miniinv 2012; 7 (3): 220-223. 